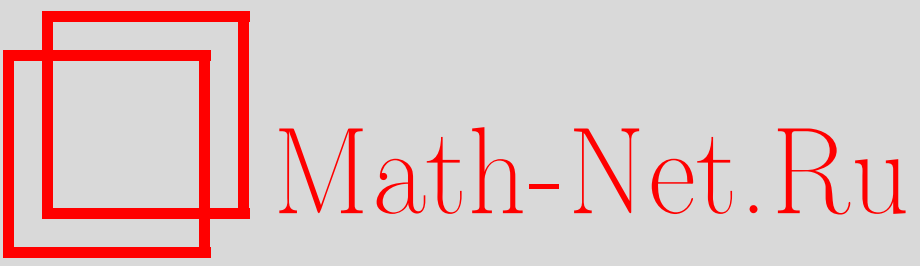

Г. Э. Абдурагимов, О существовании и единственности положительного решения краевой задачи для одного нелинейного функциональнодифференциального уравнения дробного порядка, Итоги науки и техн. Сер. Соврем. мат. и ее прил. Темат. обз., 2021, том 194, 3-7

DOI: https://doi.org/10.36535/0233-6723-2021-194-3-7

Использование Общероссийского математического портала Math-Net.Ru подразумевает, что вы прочитали и согласны с пользовательским соглашением

http://www . mathnet.ru/rus/agreement

Параметры загрузки:

IP : 54.164 .48 .24

26 апреля 2023 г., 10:20:50 


\title{
О СУЩЕСТВОВАНИИ И ЕДИНСТВЕННОСТИ ПОЛОЖИТЕЛЬНОГО РЕШЕНИЯ КРАЕВОЙ ЗАДАЧИ ДЛЯ ОДНОГО НЕЛИНЕЙНОГО ФУНКЦИОНАЛЬНО-ДИФФЕРЕНЦИАЛЬНОГО УРАВНЕНИЯ ДРОБНОГО ПОРЯДКА
}

\author{
() 2021 г. $\quad$ Г. Э. АБДУРАГИМОВ
}

Посвящается отиу, доценту Э. И. Абдурагимову

\begin{abstract}
АннотАция. В статье рассматривается краевая задача для одного нелинейного функциональнодифференциального уравнения дробного порядка. С помощью специальных топологических средств доказано существование единственного положительного решения рассматриваемой задачи.
\end{abstract}

Ключевые слова: положительное решение, краевая задача, конус, функция Грина.

\section{ON THE EXISTENCE AND UNIQUENESS \\ OF A POSITIVE SOLUTION TO A BOUNDARY-VALUE PROBLEM FOR A NONLINEAR FRACTIONAL-ORDER FUNCTIONAL DIFFERENTIAL EQUATION}

\section{(c) 2021 G. E. ABDURAGIMOV}

\begin{abstract}
In this paper, we consider a boundary-value problem for one nonlinear functional differential equation of fractional order. Using special topological means, we prove the existence of a unique positive solution to the problem considered.

Keywords and phrases: positive solution, boundary-value problem, cone, Green's function.

AMS Subject Classification: $34 \mathrm{~K} 10$
\end{abstract}

1. Введение. Вопросам исследования разрешимости функционально-дифференциальных уравнений посвящено достаточно большое количество работ (см. [1,4-13]). Практически во всех вышеупомянутых работах, в основном, рассматриваются вопросы существования положительных решений, их поведения, асимптотики и т.д., причем естественным орудием исследования являются методы функционального анализа, основанные на использовании полуупорядоченных пространств, теория которых связана с именами Ф. Рисса, М. Г. Крейна, Л. В. Канторовича, Г. Фрейденталя, Г. Биркгофа и др. В последующем методы исследования положительных решений операторных уравнений были развиты М. А. Красносельским и его учениками Л. А. Ладыженским, И. А. Бахтиным, В. Я. Стеценко, Ю. В. Покорным и др.

Работ, посвященных исследованию существования и единственности положительного решения краевой задачи для нелинейных функционально-дифференциальных уравнений дробного порядка мало. В настоящей работе предпринята попытка устранить данный пробел. На основе методов 
фунционального анализа с помощью специальных топологических средств доказано существование и единственность положительного решения краевой задачи для одного нелинейного функционально-дифференциального уравнения дробного порядка. Полученные результаты являются продолжением исследований автора, посвященных данной тематике.

2. Постановка задачи и основные результаты. Обозначим через $C$ пространство $C[0,1]$, через $\mathbb{L}_{p}(1<p<\infty)$ - пространство $\mathbb{L}_{p}(0,1)$ и через $\mathbb{W}^{2}$ - пространство вещественных функций, определенных на $[0,1]$ с абсолютно непрерывной производной.

Рассмотрим краевую задачу

$$
\begin{aligned}
& D_{0+}^{\alpha} x(t)+f(t,(T x)(t))=0, \quad 0<t<1, \\
& x(0)=0, \quad x(1)=0,
\end{aligned}
$$

где $\alpha \in(1,2]$ - действительное число, $D_{0+}^{\alpha}$ - дробная производная Римана-Лиувилля (см. [12]), $T: C \rightarrow \mathbb{L}_{p}(1<p<\infty)$ - линейный положительный непрерывный оператор, здесь функция $f(t, u)$ неотрицательна и удовлетворяет условию Каратеодори.

Под положительным решением задачи (1)-(2) будем понимать функцию $x \in \mathbb{W}^{2}$, положительную в интервале $(0,1)$, удовлетворяющую почти всюду уравнению (1) и краевым условиям $(2)$.

Рассмотрим эквивалентное задаче (1)-(2) интегральное уравнение

$$
x(t)=\int_{0}^{1} G(t, s) f(s,(T x)(s)) d s, \quad 0 \leqslant t \leqslant 1,
$$

где

$$
G(t, s)= \begin{cases}\frac{[t(1-s)]^{\alpha-1}-(t-s)^{\alpha-1}}{\Gamma(\alpha)}, & \text { если } 0 \leqslant s \leqslant t, \\ \frac{[t(1-s)]^{\alpha-1}}{\Gamma(\alpha)}, & \text { если } t \leqslant s \leqslant 1 .\end{cases}
$$

Предположим, что $f(t, u)$ удовлетворяет условию

$$
f(t, u) \leqslant a_{1}(t)+b_{1} u^{p / q}, \quad t \in[0,1], \quad u \geqslant 0,
$$

где $b_{1}>0, a_{1}(t) \in \mathbb{L}_{q}(1<q<\infty)$ - положительная функция.

В операторной форме уравнение (3) можно переписать в виде

$$
x=G N T x,
$$

где $N: \mathbb{L}_{p} \rightarrow \mathbb{L}_{q}$ - оператор Немыцкого, $G: \mathbb{L}_{q} \rightarrow C$ - оператор Грина.

Оператор $A$ определяемый равенством

$$
(A x)(t)=\int_{0}^{1} G(t, s) f(s,(T x)(s)) d s, \quad 0 \leqslant t \leqslant 1,
$$

действует в пространстве неотрицательных непрерывных функций, вполне непрерывен (см. [3, c. 161]) и оставляет инвариантным конус $\tilde{K}$ неотрицательных функций $x(t)$ пространства $C$.

Пусть

$$
f(t, u) \geqslant a_{0}(t)+\psi(u), \quad t \in[0,1], \quad u \geqslant 0,
$$

где $a_{0} \in \mathbb{L}_{q}$ - положительная функция, $\psi(u)$ - неотрицательная функция.

Введем обозначения:

$$
\Omega_{-}=\left\{x \in \tilde{K}:\|x\|_{C} \leqslant r_{-}\right\}, \quad \Omega_{+}=\left\{x \in \tilde{K}:\|x\|_{C} \leqslant r_{+}\right\}, \quad \Omega=\bar{\Omega}_{+} \backslash \Omega_{-},
$$

где

$$
r_{-}=\frac{1}{4^{\alpha-1} \Gamma(\alpha)} \int_{1 / 4}^{3 / 4} a_{0}(s)(1-s)^{\alpha-1} d s, \quad r_{+}=\left(\frac{4^{\alpha-1} \Gamma(\alpha)}{(p / q)\left\|a_{1}\right\|_{\mathbb{L}_{q}}}\right)^{q /(p-q)} .
$$

Здесь $\tau$ - норма оператора $T: C \rightarrow \mathbb{L}_{p}$. 
Теорема 1. Предположим, что $T: C \rightarrow \mathbb{L}_{p}$ - положительный на конусе $\tilde{K}$ оператор (см. [2, с. 59], выполнены неравенства (4) и (5), а также условия

$$
p>q>1, \quad \frac{\left\|a_{1}\right\|_{\mathbb{L}_{q}}\left(b_{1} \tau^{p / q}\right)^{p / q-1}}{\left(4^{\alpha-1} \Gamma(\alpha)\right)^{p / q}} \leqslant \frac{(p / q-1)^{p / q-1}}{(p / q)^{p / q}} .
$$

Тогда краевая задача (1)-(2) имеет по крайней мере одно положительное решение $x \in \Omega$.

Доказательство. Покажем существование такого числа $r_{+}>0$, что при $x \in \tilde{K}$ и $x \in \partial \Omega_{+}$ выполняется неравенство

$$
\|A x\|_{C} \leqslant\|x\|_{C}
$$

В силу (4) имеем

$$
\begin{aligned}
\|A x\|_{C}=\max _{0 \leqslant t \leqslant 1} \int_{0}^{1} G(t, s) f(s, & (T x)(s)) d s \leqslant \int_{0}^{1} G(s, s) a_{1}(s) d s+b_{1} \int_{0}^{1} G(s, s)(T x)^{p / q}(s) d s \leqslant \\
\leqslant & \frac{\left\|a_{1}\right\|_{\mathbb{L}_{q}}}{4^{\alpha-1} \Gamma(\alpha)}+\frac{b_{1}}{4^{\alpha-1} \Gamma(\alpha)}\|T x\|_{\mathbb{L}_{p}^{p / q}}^{p /} \frac{\left\|a_{1}\right\|_{\mathbb{L}_{q}}}{4^{\alpha-1} \Gamma(\alpha)}+\frac{b_{1}}{4^{\alpha-1} \Gamma(\alpha)} \tau^{p / q}\|x\|_{\mathbb{L}_{p}}^{p / q} .
\end{aligned}
$$

Рассмотрим функцию

$$
\varphi(r)=r-\beta_{1} r^{p / q}-\beta_{2}, \quad \beta_{1}=\frac{\left\|a_{1}\right\|_{\mathbb{L}_{q}}}{4^{\alpha-1} \Gamma(\alpha)}, \quad \beta_{2}=\frac{b_{1}}{4^{\alpha-1} \Gamma(\alpha)} \tau^{p / q} .
$$

Несложно убедиться, что наибольшее значений этой функции при $r>0$ обеспечивается при

$$
r=r_{\max }=\left(\frac{1}{(p / q) \beta_{1}}\right)^{q /(p-q)} .
$$

Отсюда, в силу условия (2) теоремы, гарантирующего неотрицательность функции $\varphi$ в точке $r_{\max }$, при

$$
r_{+}=r_{\max }=\left(\frac{4^{\alpha-1} \Gamma(\alpha)}{(p / q)\left\|a_{1}\right\|_{\mathbb{L}_{q}}}\right)^{q /(p-q)}
$$

следует (6).

Найдем теперь такое число $r_{-}>0$, что $\|A x\|_{C} \geqslant\|x\|_{C}$ при $x \in \tilde{K}$ и $x \in \partial \Omega_{-}$. В силу (5) и соответствующих свойств (см. [12]) функции Грина имеем

$$
\begin{aligned}
(A x)(t)=\int_{0}^{1} G(t, s) f(s, & (T x)(s)) d s \geqslant \int_{0}^{1} \gamma(s) G(s, s) f(s,(T x)(s)) d s \geqslant \\
& \geqslant \int_{1 / 4}^{3 / 4} a_{0}(s) \gamma(s) G(s, s) d s+\int_{1 / 4}^{3 / 4} \gamma(s) G(s, s) \psi(u) d s \geqslant \\
& \geqslant \int_{1 / 4}^{3 / 4} a_{0}(s) \gamma(s) G(s, s) d s=\frac{1}{4^{\alpha-1} \Gamma(\alpha)} \int_{1 / 4}^{3 / 4} a_{0}(s)(1-s)^{\alpha-1} d s,
\end{aligned}
$$

где $\gamma(s)$ - положительная непрерывная функция (см. [12]). Положив в последнем неравенстве

$$
r_{-}=\frac{1}{4^{\alpha-1} \Gamma(\alpha)} \int_{1 / 4}^{3 / 4} a_{0}(s)(1-s)^{\alpha-1} d s,
$$

получим требуемое соотношение. 
Легко проверить, что $r_{-}<r_{+}$. Согласно согласно теореме Го-Красносельского о неподвижной точке (см. [2]) оператор $A$ имеет по крайней мере одну неподвижную точку в $\Omega$, что равносильно существованию по меньшей мере одного положительного решения $x \in \Omega$ краевой задачи (1)-(2).

Теорема 2. При выполнении условий теоремы 1 краевая задача (1)-(2) имеет единственное положительное решение $x \in \Omega$, если функиия $f(t, u)$ дифферениируема по $u$, производнал $f_{u}^{\prime}(t, u)$ возрастает по второму аргументу и

$$
\|\theta\|_{\mathbb{L}_{p^{\prime}}}<\frac{4^{\alpha-1} \Gamma(\alpha)}{\tau}
$$

где $\theta(t) \equiv f_{u}^{\prime}\left(t, r_{+}(T 1)(t)\right), 1 / p^{\prime}+1 / p=1$.

Доказательство. Пусть $x_{1}(t)$ и $x_{2}(t)$ произвольные элементы множества $\Omega, y(t)=\left|x_{1}(t)-x_{2}(t)\right|$. В силу монотонности производной $f_{u}^{\prime}(t, u)$ по второму аргументу, применяя соответствующую теорему о среднем, получим

$$
\begin{gathered}
\left\|A x_{1}-A x_{2}\right\|_{C}=\max _{0 \leqslant t \leqslant 1} \int_{0}^{1} G(t, s)\left|f_{u}^{\prime}(s,(T \tilde{x})(s))(T y)(s)\right| d s \leqslant \\
\leqslant \frac{1}{4^{\alpha-1} \Gamma(\alpha)} \int_{0}^{1}\left|f_{u}^{\prime}\left(s, r_{+}(T 1)(s)\right)\right||(T y)(s)| d s \leqslant \\
\leqslant \frac{1}{4^{\alpha-1} \Gamma(\alpha)}\|\theta\|_{\mathbb{L}_{p^{\prime}}}\|T y\|_{\mathbb{L}_{p}} \leqslant \frac{\tau}{4^{\alpha-1} \Gamma(\alpha)}\|\theta\|_{\mathbb{L}_{p^{\prime}}}\|y\|_{C},
\end{gathered}
$$

где функция $(T \tilde{x})(t)$ принимает значения промежуточные между значениями $\left(T x_{1}\right)(t)$ и $\left(T x_{2}\right)(t)$.

С учетом условия (7) теоремы на основании принципа сжатых отображений заключаем, что краевая задача (1)-(2) имеет единственное положительное решение $x \in \Omega$.

В качестве примера можно рассмотреть краевую задачу

$$
\begin{aligned}
& D_{0+}^{3 / 2} x(t)+\left(\int_{0}^{1} x(s) d s\right)^{2}+\beta=0, \quad 0<t<1, \\
& x(0)=0, \quad x(1)=0,
\end{aligned}
$$

где $\beta$ - некоторое положительное число. Нетрудно проверить, что при выполнении требования

$$
1<\beta<\frac{2 \sqrt{6} \cdot \Gamma(3 / 2)}{\sqrt{3^{3 / 2}-1}}
$$

на основании вышеприведенных теорем краевая задача (8)-(9) имеет единственное положительное решение $x \in \Omega$, где

$$
r_{-}=\frac{\beta}{24 \Gamma(3 / 2)}\left(3^{3 / 2}-1\right), \quad r_{+}=\frac{\Gamma(3 / 2)}{\beta} .
$$

В частности, при $\beta=1,01$ задача (8)-(9) на конусе $\tilde{K}$ пространства $C$ имеет единственное положительное решение $x$, удовлетворяющее условию $0,199 \leqslant\|x\|_{C} \leqslant 0,877$.

\section{СПИСОК ЛИТЕРАТУРЫ}

1. Азбелев Н. В., Максимов В. П., Симонов П. М. Функционально-дифференциальные уравнения и их приложения// Вестн. Удмурт. гос. ун-та. Мат. - 2009. - 1. - С. 3-23.

2. Красносельский M. А. Положительные решения операторных уравнений. - М.: Физматгиз, 1962.

3. Крейн С. Г. Функциональный анализ. - М.: Наука, 1972.

4. Agarwal R. P., Stanek S. Positive solutions of singular value problems for delay differential equations// Dynam. Syst. Appl. — 2007. — 16, № 4. - P. 755-770. 
5. Hong C., Yeh C., Lee C., Wong F. Existence of positive solutions for functional equations// Comput. Math. Appl. - 2000. - 40. - C. 783-792.

6. Hong C., Yeh C., Lee C., Wong F. Existence of positive solutions for higher-order functional differential equations// J. Math. Anal. Appl. - 2004. - 297, № 1. - C. 14-23.

7. $M a R$. Positive solutions for boundary value problems of functional differential equations// Appl. Math. Comput. - 2007. - 193, № 1. - P. 66-72.

8. Sun Y., Han M., Debnath L. Existence of positive periodic solutions for a class of functional differential equations// Appl. Math. Comput. - 2007. - 190, № 1. - C. 699-704.

9. Weng P., Jiang D. Existence of positive solutions for boundary value problem of second-order FDE// Appl. Math. Comput. - 1999. - 37, № 10. - C. 1-9.

10. Wong F. H., Wang S. P., Chen T. G. Existence of positive solutions for second order functional differential equations// Comput. Math. Appl. - 2008. - 10, № 10. - P. 2580-2587.

11. Yin F., Fugi F., Li Y. The existence of positive solutions for the quasilinear functional delay differential equations// J. Math. Stud. - 2002. - 35, № 4. - C. 364-370.

12. Zhanbing B., Haishen L. Positive solutions for boundary value problem on nonlinear fractional differential equation// J. Math. Anal. Appl. - 2005. - 311, № 2. - C. 495-505.

13. Zima M. On positive solutions of functional differential equations in Banach spaces// J. Inequalities Appl. -2000 . - 6, № 3. - P. 359-371.

Абдурагимов Гусен Эльдерханович

Дагестанский государственный университет, Махачкала

E-mail: gusen_e@mail.ru 